\title{
Creencias, opiniones y actitudes del personal de salud del Hospital de Clínicas no especialistas en salud mental sobre personas con trastornos psicosociales, 2020
}

\section{Beliefs, opinions and attitudes of the health staff of the hospital of clinics non-specialist in mental health on people with psychosocial disorders, 2020}

\author{
Julio César Ferreira Almada ${ }^{a}$
}

\begin{abstract}
Resumen
En conformidad con la estrategia de la OMS para erradicar las actitudes estigmatizadoras, reconocer las creencias, opiniones y actitudes no adecuadas de aquellos que se ven directamente vinculados con la atención en salud, es de suma importancia con el fin de buscar las medidas adecuadas para poder revertirlas en aras de una mejor calidad de atención integral. El objetivo del presente estudio fue determinar creencias, opiniones y actitudes del personal de salud no especialista en salud mental del Hospital de Clínicas sobre personas con trastornos psicosociales. Es un estudio observacional, descriptivo y prospectivo de corte transversal, orientado al personal de salud no especialista en salud mental que desempeña funciones asistenciales en el Hospital de Clínicas, utilizando el cuestionario AQ-27. Entre los resultados, 83 profesionales, media de 35,1 $\pm 8,5$ años. De sexo femenino el 66,3\%. Enfermería 15,7\%, Médicos: Internos 21,7\%, Residentes 25,3\%, Especialistas $18 \%$ y Subespecialistas 19,3\%. Respecto a Responsabilidad: alto $1,2 \%$, intermedio $54,2 \%$, bajo $44,6 \%$. Piedad:alto $34,9 \%$, intermedio $63,9 \%$, bajo $1,2 \%$. Irritabilidad intermedio $44,6 \%$, bajo $55,4 \%$. Peligrosidad: alto $3,6 \%$, intermedio $72,3 \%$, bajo $24,1 \%$. Miedo: $8,4 \%$, intermedio $43,4 \%$, bajo $48,2 \%$. Ayuda: alto $59 \%$, intermedio $38,6 \%$, bajo $2,4 \%$. Coacción: alto $45,8 \%$, intermedio $53 \%$, bajo 1,2\%. Segregación: alta 4,8\%, intermedio $41 \%$ baja, 54,2\%. Evitación: alto 3,6\%, intermedio $56,6 \%$, bajo $39,8 \%$. Se identificó la presencia de creencias, opiniones y actitudes estigmatizantes en la población estudiada, por lo que resulta de vital importancia dar atención a estos factores para una mejor acogida tanto de los pacientes como de los profesionales en salud mental en los Hospitales Generales.
\end{abstract}

Palabras clave: cuestionario de atribución, estigma, trastornos psicosociales, personal de salud. a Universidad Nacional de Asunción, Facultad de Ciencias Médicas, Hospital de Clínicas, Paraguay.

Correspondencia a: Consultorio.psq.ferreira@gmail.com

Recibido:

1 de noviembre de 2020 Aceptado:

19 de noviembre de 2020

\section{(c) (i)}

Artículo publicado en acceso abierto bajo la Licencia Creative Commons.

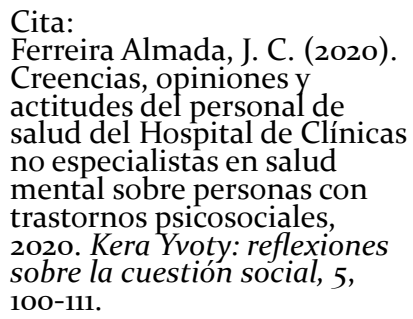


Ferreira Almada, J. C. Creencias, opiniones y actitudes del personal de salud del Hospital de Clínicas no especialistas en salud mental sobre personas con trastornos psicosociales, 2020.

\begin{abstract}
In conformity with the WHO strategy to eradicate attitudes stigmatizing, recognizing the inappropriate beliefs, opinions and attitudes of those that are directly linked to health care, it is of utmost importance with the end of finding the appropriate measures to be able to reverse them in the interests of a better quality of comprehensive care. The objective of the present study was to determine the beliefs, opinions and attitudes of non-specialist health personnel in mental health from the Hospital de Clínicas on people with psychosocial disorders. It is an observational, descriptive and prospective cross-sectional study, aimed at non-mental health specialist health personnel who perform functions care in the Hospital de Clínicas, using the AQ-27 questionnaire. Among the results, 83 professionals, mean of $35.1 \pm 8.5$ years. $66.3 \%$ were female. Nursing $15.7 \%$, Doctors: Interns $21.7 \%$, Residents $25.3 \%$, Specialists $18 \%$ and Subspecialists 19.3\%. Regarding Responsibility: high $\mathbf{1 . 2} \%$, intermediate $54.2 \%$, low $44.6 \%$. Piety: high $34.9 \%$, intermediate $63.9 \%$, low $1.2 \%$. Intermediate irritability $44.6 \%$, low $55.4 \%$.Danger: high $3.6 \%$, intermediate $72.3 \%$, low 24.1\%. Fear: $8.4 \%$, intermediate $43.4 \%$, low $48.2 \%$. Help: high $59 \%$, intermediate $38.6 \%$, low $2.4 \%$. Duress: high $45.8 \%$, intermediate $53 \%$, under $1.2 \%$. Segregation: high $4.8 \%$, intermediate $41 \%$ low, $54.2 \%$. Avoidance: high 3.6\%, intermediate $56.6 \%$, low $39.8 \%$. The presence of stigmatizing beliefs, opinions and attitudes was identified in the population studied, so it is vitally important to pay attention to these factors for a better reception of both patients and mental health professionals in General Hospitals.
\end{abstract}

Keywords: attribution questionnaire, stigma, psychosocial disorders, health personnel.

\section{Introducción}

Las creencias, opiniones y actitudes acerca de las personas con trastornos psicosociales pueden resultar estigmatizantes, cuando son basadas en conocimientos errados y estereotipos, los cuales pueden finalmente llevar a actos discriminatorios. El estigma abarca varias dimensiones y niveles: público, institucional, familiar e incluso el autoestigma.

El concepto integral de salud abarca sin duda la salud mental, por lo que es responsabilidad tanto de los Estados como de la sociedad civil y de los organismos internacionales facilitar políticas, planes de acción y estrategias orientadas a la promoción, prevención, tratamiento, rehabilitación y recuperación de las personas con trastornos psicosociales. Así mismo debe orientarse a erradicar los factores que interfieran en dichos procesos y en la integración y desarrollo personal y social de dichas personas.

En nuestro país, en conformidad con la estrategia de la OMS para erradicar las actitudes estigmatizadoras frente a las personas con trastornos psicosociales y las consecuencias del mismo, se debe orientar a la desinstitucionalización y descentralización de los servicios de salud mental.

En este contexto, la importancia de reconocer las creencias, opiniones y actitudes estigmatizadoras de aquellos que se ven directamente vinculados con la atención en salud en general y en salud mental en específico, teniendo en cuenta la tendencia actual de asimilar los servicios de Psiquiatría dentro de los Hospitales Generales, ydebido queen losúltimos 5 años no se han publicado estudios nacionales al respecto, se vuelve de importancia a fin de tomar medidas adecuadas para revertirlas en aras de una mejor calidad de atención integral.

El objetivo general fue determinar creencias, opiniones y actitudes del personal de salud no especialista en salud mental del Hospital de Clínicas sobre personas con trastornos psicosociales a partir del Cuestionario de Atribución-27 
Ferreira Almada, J. C. Creencias, opiniones y actitudes del personal de salud del Hospital de Clínicas no especialistas en salud mental sobre personas con trastornos psicosociales, 2020.

(AQ-27) 2020. En este sentido se definieron como objetivos específicos: describir las características sociodemográficas de la población de estudio, describir las creencias y opiniones en las dimensiones medidas por el Cuestionario de Atribución-27 (AQ-27) respecto a las personas con trastornos psicosociales en la población de estudio e identificar las actitudes en las dimensiones medidas por el Cuestionario de Atribución-27 (AQ-27) respecto de las personas con trastornos psicosociales en la población de estudio.

\subsection{Estigma, trastornos psicosociales y Salud Mental}

En el año 2001 la OMS creó el Programa Mundial de Acción en Salud Mental, dando un mensaje claro a la opinión pública, gobernantes y profesionales de la salud pública: "La salud mental, demasiado tiempo descuidada, es fundamental para el bienestar general de las personas, las sociedades y los países, y exige nuevos planteamientos en todos los frentes". Durante la vigencia planteada para esta iniciativa, de cinco años, se tuvo el propósito de establecer alianzas estratégicas para aumentar la capacidad de los países para enfrentar en forma integral la estigmatización y la carga de los trastornos mentales (OMS, 2001).

La publicación de la OMS "Plan de Acción sobre Salud Mental 2013-2020" recalca que situaciones de carencia de hogar y encarcelación inadecuada son mucho más frecuentes entre las personas con trastornos psicosociales que en la población general lo que aumenta su marginación y vulnerabilidad. La estigmatización y la discriminación de las personas con trastornos psicosociales las hacen susceptibles a sufrir violaciones de los derechos humanos, siendo negados sus derechos económicos, sociales y culturales. Se les impone restricciones al trabajo y a la educación, así como a los derechos reproductivosyal derechodegozardel grado más alto posible de salud. Pueden llegar a sufrir de condiciones de vida inhumanas y poco higiénicas, maltratos físicos y abusos sexuales, falta de atención y prácticas terapéuticas nocivas y degradantes en los centros sanitarios (OMS, 2013).

A pesar de los cambios en varios aspectos de la práctica médica, los Hospitales Generales siguen teniendo un papel principal en el ejercicio de la profesión. En la actualidad, la complejidad de recursos humanos y tecnológicos, hacen que pacientes de diversa índole en cuanto a posibilidades diagnósticas y de manejo en prácticamente todas las especialidades y subespecialidades las cuales a pesar de tener unidades propias, se relacionan de forma constante y dinámica. La apertura de unidades de Psiquiatría en dichos Hospitales y la aparición de la Psiquiatría de enlace en los años 60 permitió una interacción más cercana y productiva con sus pares (Alarcón, 2017).

Hay ciertamente enfermedades médicas sobre las que convergen actitudes de estigma, pero esta es varias veces más evidente cuando se trata de entidades Psiquiátricas, mucho más manifiesta en la Psiquiatría de Enlace. Todos los implicados en este escenario, siendo el paciente el centro del mismo, incluyendo familiares, personal de blanco, administrativo y de otra índole, interaccionan una y otra vez, poniéndose en evidencia actitudes estigmatizadoras en muchas formas como bromas o comentarios, indirectas, actitudes, rechazo, temor (Alarcón, 2017).

El estigma empeora la calidad de vida afectando negativamente las oportunidades de educación e inserción laboral, interfiriendo con los esfuerzos de rehabilitación, perturbando la vida en familia, empobreciendo y debilitando la red social de soporte. Las personas con trastornos psicosociales permanecen asociadas con estereotipos negativos tales como: violencia y peligrosidad. Mayor estigma se adjudica aquellos trastornos por los cuales las personas son consideradas como responsables o culpables de haberlas adquirido. Las personas que tienen un episodio psicótico agudo son todavía 
percibidas como agresivas, incontrolables, poco inteligentes, impredecibles e inducen temor o miedo, por lo que son susceptibles de rotulación, exclusión y discriminación severa (Pedersen, 2005).

El estigma de los trastornos psicosociales es tal vez el factor más significativo que influye negativamente en el proceso de búsqueda terapéutica y de rehabilitación, interfiriendo con el acceso a tratamientoyacatamientodelasperspectivas médicas, y obstaculizando la vuelta a una vida normal y efectiva reintegración social. Contribuye significativamente al sufrimiento individual y colectivo, el cual puede empeorar aún más la evolución y pronóstico de la enfermedad e interfiere con el uso oportuno de los servicios de salud, el itinerario de búsqueda de cuidado y tratamiento; y de esta manera afectando el curso de la enfermedad y los resultados del tratamiento (Pedersen, 2005).

El estigma percibido conduce a ocultar o disfrazar los síntomas, lo que implica el retraso de un diagnóstico oportuno y la postergación indefinida del tratamiento, a veces con consecuencias muy serias para el curso y pronóstico de la enfermedad mental severa y persistente (Pedersen, 2005).

Se espera que los profesionales de la salud estén libres de estigma en relación a las personas con trastornos psicosociales, eso debido a que trabajan directamente con éstas y poseen conocimiento científico sobre salud mental. Además, se sabe que son formadores de opinión, lo que resulta en el hecho de que sus creencias son tomadas con alta confiabilidad. Se considera que sus opiniones son altamente divulgadas $y$ pueden tener un efecto multiplicador en la población en general y entre otros profesionales (Piotto, 2019).

Diversos estudios demuestran que los profesionales de salud en general y los profesionales de salud mental poseen ideas negativas en relación a las personas con trastornos mentales. Estos profesionales poseen actitudes negativas con relación a las personas con trastornos psicosociales y son menos optimistas sobre los resultados del tratamiento, en comparación con el público en general. Estas ideas negativas y estigmatizantes están, probablemente, relacionadas con experiencias del personal de salud con usuarios con episodios agudos de trastornos mentales. Pero tales influencias no pueden justificar actitudes discriminatorias, además de no ser posible tolerar que esos profesionales ignoren las necesidades explícitas de los usuarios y diseminen ideas repletas de estigma (Piotto, 2019).

Estudios recientes en relación a la actitud de profesionales de blanco frente a las personas con trastornos psicosociales, demuestran que persisten actitudes negativas, relacionándolos con conductas impredecibles, violencia, incurabilidad, lo que genera en los profesionales sentimientos de temor, incomodidad y rechazo (Santiago et al., 2016; Obert, 2017).

En una publicación realizada en el mayo de 2016, que realiza un análisis de las políticas públicas de salud mental vigentes en Sudamérica, resalta que en Paraguay el personal de atención primaria de la salud tiene poca formación en salud mental, loque representa una barrera para la atención de los pacientes con trastornos psicosociales, además la vinculación intersectorial es deficiente requiriéndose apoyo financiero y político para soslayar los déficit, entre los cuales es de gran importancia el acceso desigual a los servicios de salud mental a lo largo del país. A pesar de que se dispone de medicación psicotrópica, solo una minoría de la población puede acceder a ésta de forma gratuita. Se menciona también que existen políticas y planes de salud mental, pero que éstos no son considerados en la distribución los recursos económicos del estado (Henao et al., 2016).

En un artículo que busca describir la implementación de las políticas nacionales de salud mental del Paraguay, a través de las experiencias de usuarios y personal del Hospital Psiquiátrico, refiriéndose al derecho de los usuarios a ser atendidos y tratados en sus comunidades, la 
Ferreira Almada, J. C. Creencias, opiniones y actitudes del personal de salud del Hospital de Clínicas no especialistas en salud mental sobre personas con trastornos psicosociales, 2020.

centralización de la atención en el Hospital Psiquiátricoaíslaalosusuariosdesumedio, lo que genera mayor discapacidad psicosocial y se generan condiciones desfavorables que ponen en riesgo los derechos humanos de los mismos. De esta manera resalta la importancia de la descentralización y la "desmanicomialización". Este último concepto abarca la desmanicomialización "cultural, edilicia y presupuestaria" favoreciendo la implementación de modelos de servicios ambulatorios con base comunitaria. Sin embargo, la revisión realizada del "Directorio de Servicios de Atención a la salud mental en la Red Integrada e Integral de Servicios de Salud" en el año 2015, no se encontraron registros de psiquiatras en los departamentos de San Pedro, Canindeyú, Pte. Hayes, Alto Paraguay y Boquerón, haciendo notar que en estas comunidades no había acceso a especialistas en Psiquiatría. Concluyendo en este artículo resaltan la necesidad de descentralizar la atención tanto a nivel geográfico como de recursos, proponiendo la implementación de unidades especializadas de salud mental en centros asistenciales y hospitales, o desde centros de atención comunitaria (Pacheco y Hamuy, 2017).

\subsection{Cuestionario de Atribución-27}

El diseño de intervenciones para mejorar la salud mental a nivel internacional incluye entre sus objetivos la formulación de intervenciones para erradicar el estigma. Para la evaluación de la efectividad de estas intervenciones, es necesario contar con instrumentos válidos y confiables.

Uno de los instrumentos utilizados para valorar el estigma hacia personas con trastornos psicosociales es el Cuestionario de Atribución-27, que es una herramienta de evaluación autoadministrada basada en la teoría de la atribución y fue desarrollada en los Estados Unidos por Corrigan et al. (2014); Sastre Rus et al. (2019), Ochoa et al. (2016).

Se les pide a los encuestados que califiquen su nivel de conformidad con afirmaciones basadas en una viñeta hipotética sobre un hombre con esquizofrenia (Sastre Rus et al., 2019; Ochoa et al., 2019).

Consta de 27 ítems con afirmaciones relacionadas al caso hipotético. Cada elemento se clasifica, en una escala de respuesta tipo Likert de 9 puntos, que va de 1 (nada) a 9 (mucho). Las 27 preguntas se agrupan en nueve factores, cada uno compuesto por tres preguntas, por lo que cada factor tiene un rango de puntuación total de 3 a 27 puntos. A mayor puntuación, mayor será el valor de ese factor para la persona. Una vez sumados los ítems de cada factor se los clasifica en los siguientes niveles: Puntuación Alta, con puntajes mayores de 20; Puntuación Intermedia con puntajes entre 10 y 20 y Puntuación baja con puntajes menores de 10. (Sastre Rus, et al., 2019; Ochoa et al., 2016).

Los nueve factores son: responsabilidad personal (las personas tienen control y son responsables de su enfermedad mental y síntomas relacionados); enojo/irritabilidad (se culpa a los pacientes con enfermedades mentales por sus condiciones y les causa irritación y rabia); lástima/piedad (simpatía porque la gente se siente abrumada por su enfermedad); ayuda (disposición a prestar asistencia a personas con enfermedades mentales); peligrosidad (las personas con enfermedades mentales son impredecibles y pueden ser perjudiciales para ellas mismas y para los demás); miedo (las personas con enfermedades mentales deben ser temidos porque son peligrosos); evitación (manténgase alejado de personas con enfermedades mentales); segregación (las personas con enfermedades mentales deben estar aisladas de la comunidad); y coerción/ coacción (obligar a las personas a la toma de medicamentos $\mathrm{u}$ otros tratamientos). (Sastre Rus et al., 2019; Ochoa, et al., 2016).

Los elementos relacionados con la responsabilidad, peligrosidad, miedo, ira, coerción, segregación y evitación pueden asociarse con conductas discriminatorias en contraste con la ayuda y la piedad. 
Estos factores son construcciones clave del modelo social cognitivo del estigma de Corrigan, basado en la teoría de la atribución, que sostiene que la conducta está determinada por un proceso cognitivo-emocional: las personas hacen atribuciones sobre la causa y la capacidad de control de una persona hacia una enfermedad lo que conduce a inferencias sobre la responsabilidad. Estas inferencias conducen a reacciones emocionales como ira o lástima que afectan la probabilidad de comportamientos ya sean coercitivos, de evitación o de ayuda. Así, si se considera que la persona no es responsable de su enfermedad, esto lleva a una respuesta afectiva de lástima, la cual lleva a la reacción comportamental de ayuda; al contrario no se proporcionará ayuda si se considera a la persona responsable de su enfermedad. También desarrolló el modelo de peligrosidad, según el cual se realiza una atribución personal sobre la peligrosidad de la persona con enfermedad, esto desencadena miedo como reacción emocional y lleva a conductas de evitación. (Sastre Rus et al., 2019; Ochoa et al., 2016).

La escala AQ-27 ha mostrado buenos niveles de consistencia interna con alfa de Cronbach que van desde 0,7 a 0,96. En la bibliografía se encuentran versiones validadas en distintos idiomas entre ellos portugués (Oliveira et al., 2020.), italiano (Pingani et al., 2011), español (Vallecillo Chaves, 2016; Bolivar-Paredes \& VillanuevaRuska, 2017), versión turca (Akyurek et al., 2019).

\section{Metodología}

El diseño del trabajo es un estudio observacional, descriptivo y prospectivo de corte transversal, cuya muestreo es no probabilístico de casos consecutivos. La población enfocada ha sido el personal de salud no especialista en salud mental que desempeña funciones asistenciales en Hospitales Generales y la población accesibleel personal de salud no especialista en salud mental que desempeña funciones asistenciales en el Hospital de Clínicas que cumplan los criterios de inclusión y que se encuentren en el marco temporal de la investigación.

En cuanto al marco temporal, el tiempo establecido ha sido desde Agosto a Octubre de 2020. Los criterios de inclusión han sido: personal de salud no especialista en salud mental que desempeña funciones asistenciales en el Hospital de Clínicas de ambos sexos, mayores de edad y los criterios de exclusión han sido profesionales que realizan funciones administrativas, los que no accedieron a participar del estudio y aquellos cuestionarios que no estén completos o no fueron completados adecuadamente.

Para la captación de la información pertinente de las variables en estudio se proporcionó a los sujetos una encuesta digital elaborada en la plataforma de Formularios de Google ${ }^{\circledR}$ que fue distribuido por medios digitales. Las variables han sido los datos sociodemográficos y de filiación, incluyendo edad (en años), sexo (femenino, masculino); nivel educativo y área de formación en Salud: Enfermería (Técnico, Auxiliar, Licenciado), Médico (Interno, Residente, Especialista, Subespecialista). Actitudes hacia personas con trastornos psicosociales considerando las dimensiones evaluadas por el Cuestionario Estandarizado Autoaplicado AQ-27 que consta de 9 factores (Responsabilidad, Piedad, Irritabilidad, Peligrosidad, Miedo, Ayuda, Coacción, Segregación y Evitación), cada uno de ellos conformados por 3 ítems los cuales se responden en una escala tipo Likert de 1 a 9.

Como instrumento se utilizó una encuesta cerrada para medir las variables sociodemográficas y de filiación. Para la recolección de datos acerca de la actitud de los profesionales de la salud hacia pacientes con trastornos psicosociales, se empleó el cuestionario AQ-27, de 27 ítems de opción excluyente que está basado en encuestas utilizadas y validadas en la literatura. Se confeccionó una base de datos electrónica en la que fueron cargados los datos que cumplieron los criterios de inclusión para 
Ferreira Almada, J. C. Creencias, opiniones y actitudes del personal de salud del Hospital de Clínicas no especialistas en salud mental sobre personas con trastornos psicosociales, 2020.

Tabla 1. Datos sociodemográficos del Personal de salud encuestado ( $n=83$ )

\begin{tabular}{lcc}
\hline \multicolumn{1}{c}{ Variable } & Frecuencia & Porcentaje \\
\hline Sexo & & \\
$\quad$ Hombre & 28 & 33,7 \\
$\quad$ Mujer & 55 & 66,3 \\
Nivel educativo y área de formación en salud & 13 & 15,7 \\
$\quad$ Lic en Enfermería & 18 & 21,7 \\
Médico Interno & 21 & 25,3 \\
Médico Residente & 15 & 18 \\
Médico con Especialidad & 16 & 19,3 \\
$\quad$ Médico con Subespecialidad & & \\
\hline
\end{tabular}

su posterior análisis estadístico. Se realizó una prueba piloto en los servicios no incluidos en la población de estudio.

En cuanto a las mediciones, se mencionan: - Sexo: variable cualitativa nominal dicotómica, - Edad: variable cuantitativa discreta, - Nivel educativo y área de formación en salud: variable cualitativa ordinal politómica, - Ítems del Cuestionario de Atribución-27: variable cuasi-cuantitativa. El tamaño de muestra fue calculado con un intervalo de confianza del $95 \%$ y margen de error del $5 \%$, proporcionando un número mínimo de 334 muestras de un universo de 2500 personales de salud que desempeñan funciones asistenciales en el Hospital de Clínicas.

Los datos fueron cargados en una planilla de datos electrónicos para luego ser procesados con el paquete estadístico EpiInfoversión 7.2. Las variables categóricas son resumidas en forma de tablas de frecuencias, las cuantitativas con medidas de tendencia central y de dispersión.

El estudio fue aprobado por la Dirección de investigaciones de la Facultad de Ciencias Médicas de la Universidad Nacional de Asunción. Todos los participantes respondieron un consentimiento informado incluido al inicio del cuestionario digital, en el cual se detallaron los objetivos y el alcance del estudio, así como datos relevantes en cuanto a la confidencialidad y anonimato de los datos contenidos en la encuesta. Los datos fueron utilizados para cumplir con los objetivos de la investigación y se respetaron los principios de ética aplicados a la investigación, incluidos los principios de autonomía, beneficencia y no maleficencia y el de justicia.

\section{Resultados}

Se incluyeron 83 Profesionales de la salud con edades comprendidas entre los 24 y 6 o años con una media de $35,1 \pm 8,5$ años. El 66,3\% corresponde al sexo femenino.

En cuanto al nivel educativo y el área de formación en salud corresponden a Lic en Enfermería el 15,7\%, Médico Interno el 21,7\%, Médico Residente el 25,3\%, Médico con Especialidad el $18 \%$ y Médico con Subespecialidad el 19,3\% delos encuestados. Estos datos sociodemográficos se pueden ver con detalle en la Tabla 1.

En relación a los aspectos considerados en el cuestionario AQ-27 sobre las creencias, opiniones y actitudes de los profesionales de salud no especialistas en salud mental acerca de personas con trastornos psicosociales se encontró dentro del parámetro de responsabilidad: nivel alto el 1,2\%, intermedio el $54,2 \%$ y bajo el $44,6 \%$ delos encuestados. En el factor denominado piedad: nivel alto $34,9 \%$, intermedio el $63,9 \%$ y bajo el $1,2 \%$. En irritabilidad se encontró un nivel intermedio en el 44,6\% y bajo en el 55,4\%. En el parámetro de peligrosidad se encontraron con un nivel alto en el 3,6\%, intermedio en el $72,3 \%$ y bajo en el $24,1 \%$ del total. En el parámetro miedo los resultados fueron: nivel alto en el $8,4 \%$, intermedio en el $43,4 \%$ y bajo en el $48,2 \%$. En ayuda se encontró un nivel alto 
Ferreira Almada, J. C. Creencias, opiniones y actitudes del personal de salud del Hospital de Clínicas no especialistas en salud mental sobre personas con trastornos psicosociales, 2020.

Tabla 2. Creencias, opiniones y actitudes frente a personas con trastornos psicosociales por parte del personal de salud no especialista en salud mental, parámetros analizados por el cuestionario AQ-27

\begin{tabular}{|c|c|c|}
\hline Variable & Frecuencia & Porcentaje \\
\hline \multicolumn{3}{|c|}{ Responsabilidad } \\
\hline Alta & 1 & 1,2 \\
\hline Intermedia & 45 & 54,2 \\
\hline Baja & 37 & 44,6 \\
\hline \multicolumn{3}{|l|}{ Piedad } \\
\hline Alta & 29 & 34,9 \\
\hline Intermedia & 53 & 63,9 \\
\hline Baja & 1 & 1,2 \\
\hline \multicolumn{3}{|l|}{ Irritabilidad } \\
\hline Alta & o & o \\
\hline Intermedia & 37 & 44,6 \\
\hline Baja & 46 & 55,4 \\
\hline \multicolumn{3}{|l|}{ Peligrosidad } \\
\hline Alta & 3 & 3,6 \\
\hline Intermedia & 60 & 72,3 \\
\hline Baja & 20 & 24,1 \\
\hline \multicolumn{3}{|l|}{ Miedo } \\
\hline Alta & 7 & 8,4 \\
\hline Intermedia & 36 & 43,4 \\
\hline Baja & 40 & 48,2 \\
\hline \multicolumn{3}{|l|}{ Ayuda } \\
\hline Alta & 49 & 59 \\
\hline Intermedia & 32 & 38,6 \\
\hline Baja & 2 & 2,4 \\
\hline \multicolumn{3}{|l|}{ Coacción } \\
\hline Alta & 38 & 45,8 \\
\hline Intermedia & 44 & 53 \\
\hline Baja & 1 & 1,2 \\
\hline \multicolumn{3}{|l|}{ Segregación } \\
\hline Alta & 4 & 4,8 \\
\hline Intermedia & 34 & 41 \\
\hline Baja & 45 & 54,2 \\
\hline \multicolumn{3}{|l|}{ Evitación } \\
\hline Alta & 3 & 3,6 \\
\hline Intermedia & 47 & 56,6 \\
\hline Baja & 33 & 39,8 \\
\hline
\end{tabular}


Ferreira Almada, J. C. Creencias, opiniones y actitudes del personal de salud del Hospital de Clínicas no especialistas en salud mental sobre personas con trastornos psicosociales, 2020.

en el $59 \%$, intermedio en el 38,6 y bajo en el 2,4\%. En coacción los hallazgos fueron: alto en el $45,8 \%$, intermedio en $53 \%$ y baja en $1,2 \%$. En segregación los resultados obtenidos fueron: alta en el 4,8\%, intermedio en el $41 \%$ y baja en el $54,2 \%$. Finalmente en el parámetro denominado evitación se encontró un nivel alto en el $3,6 \%$, intermedio en el $56,6 \%$ y bajo en el $39,8 \%$.

En la Tabla 2 se pueden observar con detalle los resultados de los parámetros estudiados a partir del cuestionario AQ-27.

\section{Discusión}

El presente estudio se realizó con los objetivos de determinar las creencias, opiniones y actitudes del personal de salud no especialista en salud mental sobre personas con trastornos psicosociales, enfocándose a la población que desempeña funciones asistenciales en el Hospital de Clínicas.

La distribución por nivel educativo y área de formación en salud, resultó ser una muestra balanceada con porcentajes similares de cada una de las áreas de formación en salud. La participación del sexo femenino fue mayor que el sexo masculino.

En cuanto a los factores negativos relacionados con discriminación y estigma que serían responsabilidad, irritabilidad, peligrosidad, miedo, segregación y evitación se encontró predominio de niveles intermedios de atribución para la población estudiada. En el factor coacción se encontró una preponderancia de los niveles de atribución alta seguida del intermedio.

En relación a los factores relacionados con actitudes positivas, en el factor ayuda se encontró predominancia de niveles de atribución alta seguidodel nivel intermedio, en el factor piedad en cambio predominan los niveles de atribución intermedios seguido por los niveles altos.

Se encontró una relación directa moderada entre los factores de miedo y peligrosidad (coeficiente de Pearson de $0,727)$, entre miedo e irritabilidad $(0,644)$ y peligrosidad e irritabilidad $(0,623)$.

Estos resultados traen a la luz la existencia de niveles intermedios de atribución a factores negativos relacionados

Tabla 3. Coeficiente de Correlación de Pearson para los distintos parámetros analizados por el cuestionario $A Q-27$

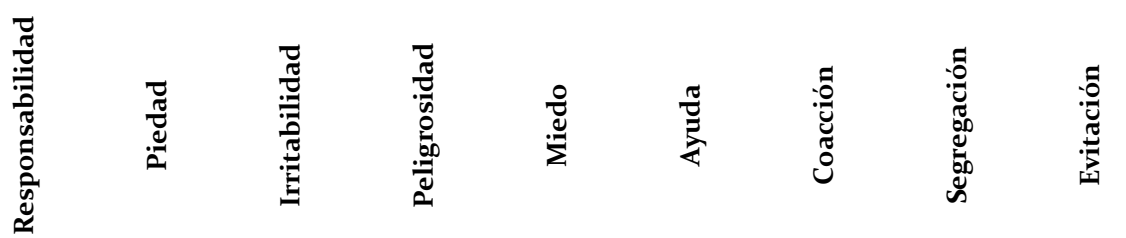

\begin{tabular}{|c|c|c|c|c|c|c|c|c|c|}
\hline Responsabilidad & 1 & & & & & & & & \\
\hline Piedad & 0,181 & 1 & & & & & & & \\
\hline Irritabilidad & 0,316 & 0,042 & 1 & & & & & & \\
\hline Peligrosidad & 0,106 & 0,116 & 0,623 & 1 & & & & & \\
\hline Miedo & 0,257 & 0,16 & $o, 644$ & 0,727 & 1 & & & & \\
\hline Ayuda & 0,118 & 0,278 & $-0,282$ & $-0,224$ & $-0,255$ & 1 & & & \\
\hline Coacción & 0,213 & 0,374 & 0,254 & 0,301 & 0,336 & 0,085 & 1 & & \\
\hline Segregación & 0,253 & o,o16 & 0,579 & 0,541 & 0,436 & $-0,106$ & 0,373 & 1 & \\
\hline Evitación & 0,099 & 0,162 & $-0,39$ & $-0,352$ & $-0,391$ & 0,546 & $-0,17$ & $-0,372$ & 1 \\
\hline
\end{tabular}


con la discriminación y estigma en una proporción importante de la población en estudio, lo que coincide con otros estudios que identifican que aún persisten actitudes, creencias y opiniones estigmatizantes en los personales de salud hacia personas con trastornos psicosociales (Piotto, 2019; Obert, 2017; Corrigan et al., 2014).

El factor negativo más resaltante fue el de coacción, lo que implica que un porcentaje importante de profesionales de la salud consideran que es necesario obligar a las personas con trastornos psicosociales a seguir un tratamiento o tomar medicación. Esto podría relacionarse con la percepción de peligrosidad e imprevisibilidad atribuida a estos pacientes, lo cual a su vez podría estar relacionado a malas experiencias con pacientes en cuadros agudos en los servicios de urgencias generales (Pedersen, 2005: Oliveira et al., 2020; Griffiths et al., 2014; Eksteen et al., 2017).

Otro factor que se ha relacionado con la persistencia de actitudes estigmatizantes es la hipótesis del contacto. Es decir que aquellos profesionales con un contacto más personal con las enfermedades mentales presentan una reducción significativa del estigma y mejora el enfoque positivo hacia los trastornos psicosociales. Esto también se ha visto con profesionales de la salud con un familiar con enfermedad mental, expresando menos actitudes estigmatizantes con puntuaciones significativamente más altas en los factores positivos (piedad y ayuda) (Oliveira et al., 2020; Griffiths et al., 2014; Eksteen et al., 2017).

Finalmente el presente trabajo, al identificar la presencia de creencias, opiniones y actitudes estigmatizantes en la población estudiada, y teniendo en cuenta que la promoción de intervenciones educativas entre otros servicios dentro de los Hospitales Generales por parte de Especialistas en Psiquiatría, reduce el estigma hacia las personas con trastornos psicosociales como hacia la misma Psiquiatría, resulta de vital importancia dar atención a estos factores para una mejor acogida tanto de los pacientes como de los profesionales en salud mental en los Hospitales Generales.

Las limitaciones del presente estudio se relacionan con la baja respuesta obtenida con los cuestionarios digitales, lo que podría deberse en parte a las medidas de prevención adoptadas por la pandemia del SARS-COV2, aunque otro factor podría estar relacionado con la dificultad de manifestar actitudes estigmatizantes siendo profesionales de la salud.

\section{Agradecimientos}

Este proyecto es financiado por el CONACYT a través del programa PROCIENCIA, con recursos del Fondo para la Excelencia de la Educación e Investigación - FEEI del FONACIDE

\section{Referencias}

Akyurek, G., Efe, A., \& Kayihan, H. (2019). Stigma and Discrimination Towards Mental Illness: Translation and Validation of the Turkish Version of the Attribution Questionnaire-27 (AQ-27-T). Community Ment Health J., 55(8), 1369-76.

Alarcón, R. D. (2017). Estigma en la práctica psiquiátrica de un Hospital General. Revista Médica Clínica Las Condes, 28(6), 818-25. https://www. sciencedirect.com/science/article/pii/ So716864017301414

Bolivar-Paredes, E, Villanueva-Ruska, A. (2015). Validación y confiabilidad del Cuestionario AQ-27 de actitudes estigmatizadoras hacia pacientes con esquizofrenia en un Hospital General - 2015. Revista de Neuro-Psiquiatría, 8o(3), 165-71. http://www.scielo.org. pe/scielo.php

Calvo, J. S. (2019). Estigma de los profesionales de enfermería de salud mental hacia las personas con trastorno mental grave. Revista Española de Enfermería de Salud Mental, (7). http:// www.reesme.com/index.php/REESMAEESME/article/view/36

Corrigan, P. W., Powell, K. J., \& Michaels, P. 
Ferreira Almada, J. C. Creencias, opiniones y actitudes del personal de salud del Hospital de Clínicas no especialistas en salud mental sobre personas con trastornos psicosociales, 2020.

J. (2014). Brief battery for measurement of stigmatizing versus affirming attitudes about mental illness. Psychiatry Research., 215(2, 466-70.

Del Olmo-Romero, F., González-Blanco, M, Sarró, S, Grácio, J, Martín-Carrasco, M, \& Martínez-Cabezón, A. C. (2019). Mental health professionals' attitudes towards mental illness: professional and cultural factors in the INTER NOS study. Eur Arch Psychiatry Clin Neurosci., 269(3), 325-39. https:// pubmed.ncbi.nlm.nih.gov/29353369/

Dickens, G. L., Lamont, E., \& Gray, S. (2016). Mental health nurses' attitudes, behaviour, experience and knowledge regarding adults with a diagnosis of borderline personality disorder: systematic, integrative literature review. Journal of Clinical Nursing., (13-14), 1848-75. https://onlinelibrary. wiley.com/doi/abs/10.1111/jocn.13202

Eksteen, H. C., Becker, P. J., \& Lippi G. (2017). Stigmatization towards the mentally ill: Perceptions of psychiatrists, preclinical and post-clinical rotation medical students. Int J Soc Psychiatry, 63(8), 782-91.

Griffiths, KM, Carron-Arthur, B, Parsons, A, Reid R. (2014). Effectiveness of programs for reducing the stigma associated with mental disorders. A meta-analysis of randomized controlled trials. World Psychiatry, 13(2), 161-75.

Henao Henao, S., Quintero Idárraga, S., Echeverri Âlvarez, J., Hernández Calle, J. A., Rivera, E., López Granada, S. (2016). Políticas públicas vigentes de salud mental en Suramérica: un estado del arte. Current public policies on mental health in South America: a state of the art. http://bibliotecadigital. udea.edu.co/handle/10495/4347

Márquez Cervantes, E. (2018). La percepción de la psiquiatría y las actitudes hacia los pacientes psiquiátricos de los estudiantes de quinto año de medicina de la Facultad de Medicina, UANL. Universidad Autónoma de Nuevo León. http://eprints.uanl.mx/17342/
Martín Balle, M. (2019). Presencia de estigma frente el Trastorno Mental Grave en estudiantes de la Universitat de les Illes Balears. http://dspace.uib. es/xmlui/handle/11201/150413

Obert, M. (2017). The Influence Of Rural Primary Care Providers Level of Stigma On Recognition of Suicidal Ideation In Patients [Theses and Dissertations]. https://commons.und. edu/theses/2302

Ochoa, S., Martínez-Zambrano, F., VilaBadia, R., Arenas, O., Casas-Anguera, E., \& García-Morales, E. (2016). Validación al castellano de la escala de estigma social: Community Attitudes towards Mental Illness en población adolescente. Revista de Psiquiatría y Salud Mental, 9(3), 150-7. https://www. sciencedirect.com/science/article/ abs/pii/S1888989115000476

Oliveira, A. M., Machado, D., Fonseca, J. B., Palha, F., Silva Moreira, P., \& Sousa, N. (2020). Stigmatizing Attitudes Toward Patients With Psychiatric Disorders Among Medical Students and Professionals. Front Psychiatry [Internet]. 2020 [citado 19 de junio de 2020];11. Disponible en: https:// www.frontiersin.org/articles/10.3389/ fpsyt.2020.00326/full?utm_source=STWT\&utm_medium=SNET\&utm campaign=ECO_FPSYT_XXXXXXXX_auto-dlvrit

Organización Mundial de la Salud, OMS. (2001). Programa Mundial de Acción en Salud Mental. https://www.who.int/ mental_health/media/en/267.pdf

Organización Mundial de la Salud, OMS. (2013).PlandeAcción sobreSaludMental 2013-2020. https://apps.who.int/iris/ bitstream/37273FFoBC2C? sequence $=1$

Pacheco, C., \& Hamuy, F. (2017). La política nacional de salud mental y los servicios de atención del hospital psiquiátrico de Asunción. Estudios Paraguayos. 26. https://epy.dreamhosters.com/index. $\mathrm{php} / \mathrm{RESPY} / \mathrm{article} / \mathrm{view} / 53 / 49$

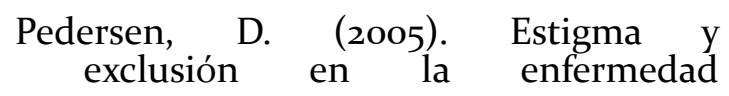


mental: Apuntes para el análisis e investigación. Revista de Psiquiatría y Salud Mental, 6, 3-14. https://www. researchgate.net/profile/Duncan Pedersen/publication/281364699_ Estigma_y_exclusion_en_la_ enfermedad_mental_Apuntes para_el_analisis_e_investigacion/ links/5613df65o8aec622440fd69a/ Es tig ma-y-exclusion-en-laenfermedad-mental-Apuntes-para-elanalisis-e-investigacion.pdf

Pingani, L., Forghieri, M., Ferrari, S., Ben-Zeev, D., Artoni, P., \& Mazzi, F. (2011). Stigma and discrimination toward mental illness: translation and validation of the Italian version of the Attribution Questionnaire-27 (AQ27-I). Social psychiatry and psychiatric epidemiology, 47, 993-9.

Piotto, R. (2019). Adaptação cultural da Mental Illness: Clinicians \' Attitudes Scale (MICA-4) para o português brasileiro [Mestrado em Enfermagem Psiquiátrica]. Universidade de São Paulo. http://www.teses.usp.br/teses/ disponiveis/22/22131/tde-12042019182911/
Santiago, H. G., Navarro, M. W., Pérez, P. L., \& Betancort, E. N. (2016). El estigma hacia personas con enfermedad mental en profesionales sanitarios del hospital general. Norte de Salud Mental, 14(55), 103-11. https://dialnet.unirioja.es/ servlet/articulo codigo $=5836868$

Sastre-Rus, M., García-Lorenzo, A., Lluch-Canut, M.-T., Tomás-Sábado, J., \& Zabaleta-Del-Olmo, E. (2019). Instruments to assess mental healthrelated stigmaamong health professionals and students in health sciences: A systematic psychometric review. Journal of Advanced Nursing., 75(9), 1838-53.

Vallecillo Chaves, N. (2016). Una aproximación psicométrica para la validación de la escala Attribution Questionnaire-27 sobre estigma social hacia las personas con enfermedad mental. https://idus.us.es/ handle/11441/45276

Wei, Y, McGrath, P, Hayden, J, Kutcher, S. (2018). The quality of mental health literacy measurement tools evaluating the stigma of mental illness: a systematic review. Epidemiology and Psychiatric Sciences, 27(5):433-62.

\section{Sobre el Autor}

Julio César Ferreira Almada

Doctor en Medicina y Cirugía de la Universidad Nacional de Asunción. Tercer año de Residencia Psiquiatría Clínica de la FCM-UNA. 